Article

\title{
Agenda Trending: Reciprocity and the Predictive Capacity of Social Networking Sites in Intermedia Agenda Setting across Topics over Time
}

Jacob Groshek ${ }^{1, *}$ and Megan Clough Groshek ${ }^{2}$

${ }^{1}$ College of Communication, Boston University, 640 Commonwealth Avenue, Boston, MA 02215, USA;

E-Mail: jgroshek@bu.edu; Tel.: +1 6173536421; Fax: +1 6173533405

${ }^{2}$ Office of Academic Services, Brandeis University, 415 South Street, Waltham, MA 02453, USA;

E-Mail: mgroshek@brandeis.edu

* Corresponding author

\section{How to Cite this Article}

Groshek, J., \& Groshek, M. C. (2013). Agenda Trending: Reciprocity and the Predictive Capacity of Social Networking Sites in Intermedia Agenda Setting across Topics over Time. Media and Communication, 1(1), 15-27.

\section{Acknowledgement}

This Article was published by Librello, Media and Communication's former publisher.

\begin{abstract}
About the Journal
Media and Communication is an international open access journal dedicated to a wide variety of basic and applied research in communication and its related fields. It aims at providing a research forum on the social and cultural relevance of media and communication processes.
\end{abstract}

www.cogitatiopress.com/mediaandcommunication

\section{Editors-in-Chief}

Professor Bradley Greenberg, Departments of Communication and Telecommunication, Information Studies and Media, Michigan State University, USA

Professor Elisabeth Klaus, Department of Communication, University of Salzburg, Austria

\section{Managing Editor}

Mr. António Vieira, Media and Communication, Cogitatio Press, Portugal 


\title{
Agenda Trending: Reciprocity and the Predictive Capacity of Social Networking Sites in Intermedia Agenda Setting across Topics over Time
}

\author{
Jacob Groshek ${ }^{1, *}$ and Megan Clough Groshek ${ }^{2}$ \\ ${ }^{1}$ College of Communication, Boston University, 640 Commonwealth Avenue, Boston, MA 02215, USA; \\ E-Mail: jgroshek@bu.edu; Tel.: +1 6173536421; Fax: +1 6173533405 \\ ${ }^{2}$ Office of Academic Services, Brandeis University, 415 South Street, Waltham, MA 02453, USA; \\ E-Mail: mgroshek@brandeis.edu \\ * Corresponding author
}

Submitted: 17 April 2013 | In revised form: 19 July 2013 | Accepted: 5 August 2013 |

Published: 23 August 2013

\begin{abstract}
In the contemporary converged media environment, agenda setting is being transformed by the dramatic growth of audiences that are simultaneously media users and producers. The study reported here addresses related gaps in the literature by first comparing the topical agendas of two leading traditional media outlets (New York Times and CNN) with the most frequently shared stories and trending topics on two widely popular Social Networking Sites (Facebook and Twitter). Time-series analyses of the most prominent topics identify the extent to which traditional media sets the agenda for social media as well as reciprocal agendasetting effects of social media topics entering traditional media agendas. In addition, this study examines social intermedia agenda setting topically and across time within social networking sites, and in so doing, adds a vital understanding of where traditional media, online uses, and social media content intersect around instances of focusing events, particularly elections. Findings identify core differences between certain traditional and social media agendas, but also within social media agendas that extend from uses examined here. Additional results further suggest important topical and event-oriented limitations upon the predictive capacity of social networking sites to shape traditional media agendas over time.
\end{abstract}

Keywords: election coverage; focusing events; Granger causality; intermedia agenda setting; social media 


\section{Introduction}

Since the seminal agenda-setting article of McCombs and Shaw [1], much has been made of the ability of media content to inform audiences what to think about rather than what to think. As originally introduced in that study, agenda setting conceptually identified that mass media-specifically local and national newspapers, national news magazines, and national television evening news broadcasts-directly shaped the public agenda by strategically highlighting specific topics, issues, and actors in coverage over others. This process of increasing salience among media audiences, begun by McCombs and Shaw's initial inquiry of presidential campaign coverage, has now been explored across explanatory dimensions such as need for orientation [2] as well as attributes in second-level agenda setting [3]. In addition, agenda setting evidence has been reported in a wide range of national contexts across diverse issues including elections, health, crime, war, and culture, among others [4].

Yet, whether dealing with election campaigns or other topics, mass media coverage shares a common feature: limited, and necessarily hierarchical, space to address a technologically limited array of issues. Agenda setting has therefore often been positioned as a technological byproduct of the gatekeeping activities of journalists and editors. On this point, Groshek [5] wrote, "because there is only so much space on the front page of a newspaper and only so much time devoted to the news on radio and television, agenda setting is unavoidable". While the Internet and the transition of media production and audiences online has ameliorated the critical aspect of available space, the presentation order and style of reporting [6], as well as the number of stories that are covered in online channels have been shown to have agendasetting effects [7], though measuring agendas at a broad topical level does introduce certain limitations.

At the intersection of media technologies and agendas is the well-documented shift towards a dynamic user-producer media environment [8]. Indeed, the notion of media "produsers" [9] has to some extent upended the norm of agenda setting running from mass media to the public $[10,11]$ in a manner that conceptually resembles Entman's [12] cascading activation model. Importantly, however, this technologically engendered transformation has not only made it possible for audiences, but also editors and journalists to easily monitor the output of multiple media organizations, regardless of time and location. By some accounts, such practices have actually been linked to increasing the homogenization of both media and public agendas, rather than diversifying them [13-15].

Therefore, topics deemed important by leading media channels, sometimes regardless of geographical region, continue to identify similar-if not identical -topics across media outlets and platforms. Numerous scholars have noted the general lack of, or decline in, diversity of news content over recent decades (see, for example, Gans [16]), and Schudson wrote, "the stories one reads in one publication are likely to bear a stronger resemblance to the stories in the next publication than they would have in the past" (as cited in Boczkowski and de Santos [14]).

Considering the somewhat paradoxical confluence of these trends, this study examines the intermedia agenda-setting influence of leading mass media on leading Social Networking Sites (SNS) [17]. Specifically, coverage from the online editions of the New York Times and CNN are separately measured over time with the most frequently shared news items on Facebook and the highest-ranked trending topics on Twitter. In so doing, this study considers not only the concepts of media homogenization and user-generated content but also the reciprocity of intermedia agenda setting across professional/de-professionalized boundaries. Crucially, this study thus advances the extant literature by modeling the time-ordered effect that social media agendas, such as those found on Facebook and Twitter can have on the agendas of leading traditional media in their online formats.

In relation to this goal, which builds upon previous findings of Meraz [11] and Song [6] in identifying Weblogs and other alternative online media as shaping media and public agendas, the literature review broadly situates the current state of agenda-setting research with regard to social networking sites. Further, the review of literature examines the import of event-driven news and immediate reporting, specifically involving politics, in both mass and social media to consider the empirical evidence of reciprocity in agenda trends.

\section{Agenda Setting from Mass Media through Social Media}

In a recent study, Meraz [11] reported that, over time, "agenda setting has matured as a theory to include a second-level agenda-setting component (attribute agenda setting), a psychological component to explain individual-level agenda-setting effects (need for orientation), an emphasis on how the media's agenda is shaped, and an explanation for the shared news agenda among different media (intermedia agenda setting)". Still, while this theoretical approach of media effects research has been analyzed at great lengths (with 567,000 topical hits on Google Scholar reported by Bennett and Iyengar [18] in 2008), found at different levels, and codified into unique typologies, agenda setting is being transformed by the dramatic growth of audiences that are simultaneously both media users and producers, notably on social networking sites.

Indeed, agenda setting is no longer conceived of as only a top-down process from (mainstream print and broadcast) media to audiences, but also as a dynamic process where, under certain conditions, citizen re- 
porting advanced in online spaces can give shape and definition to media and policy agendas among the public $[19,20]$. Accordingly, as Sayre and colleagues reported, "the Internet is at the center of this change, expanding the definition of news sources and news producers" [21]. Without question, the rise of the media "produser," as described by Bruns [9] has altered conceptions of where media agendas begin and end in relation to the public agenda. Considering the vast array of options for online media consumers to engage, share, and create with varying levels of commitment and intensity [22], it is clear that media agendas can now regularly intersect and cross amateur and professional boundaries $[11,21,23]$, which introduces a reinvigorated conception of intermedia agenda setting that requires additional examination.

Historically, intermedia agenda setting has regularly been studied across various platforms, media systems, and geographical regions $[4,24]$. Yet, one of the most confounding results in the arena of intermedia agenda setting is the continual increase in media outlets that is set in apparent contradiction with the growing cultural and thematic homogenization of the content being presented. For example, Boczkowski and de Santos examined homogenization across Argentina's print and online newspapers and found the "intensification of online updates during the day coincides with an increase in the level of content overlap in the print and online newspapers" [14]. Similarly, Groshek examined the agendas of CNN and CNN International coverage online and found that though "there were differences in which issues were most salient on CNN and CNNI, the top three categories were the same for both: Crime, Politics, and War" [5]. Though previous research on professional intermedia agenda setting has generally found some important topical cleavages, evidence suggests that the overall and thematic differences are more subtle and becoming less distinct in what has become global media culture [25-27].

Beyond this dimension of intermedia agenda setting within and across professional media, scholars have begun to examine the spill-over [24] of online, user-generated content intersecting with the agendas of professional, traditionally offline media. In one example that employed time-series analysis [11], it was found that Weblogs contributed to setting the agendas of traditional elite media. Along these conceptual and methodological lines, Sayre et al. [21] analyzed and traced the relationships of thousands of YouTube videos and professional news media coverage of Proposition 8 (a ballot measure regarding same-sex marriage) in California. There, they reported that "YouTube was leading the charge in terms of attention to Proposition 8 in 2009" and that "online outlets such as YouTube do indeed have the potential to set the agenda independently of, and even in advance of, more professional media outlets" [21].

Another recent study compared the topical differ- ences between the New York Times and Twitter [28], and found that Twitter was a viable source of what were considered entity-oriented topics with limited coverage in traditional media. Moreover, that study also found that "although Twitter users show relatively low interests in world news, they actively help spread news of important world events". Similarly, Kwak and colleagues [29] compared Twitter's trending topics to CNN headlines and Google trends. In their analysis, they identified that CNN was ahead in reporting more than half the time, compared to Twitter. However, Kwak et al. [29] also found evidence of what can be considered "focusing events" [30] in social media agendas, noting that "some news broke out on Twitter before CNN and they are of live broadcasting nature (e.g., sports matches and accidents)" [29].

Altogether, there is fairly clear evidence from previous studies that the SNSs can be important intermedia agenda-setting agents, particularly because of their capacity to quickly and easily share stories and break news as it occurs $[21,23,28,29]$. Considering the intersection of these findings with that of previous work on focusing events [30] and live-event news [31], it seems clear that agenda setting has reached a conceptual and empirical juncture [24]. Indeed, with a reinvigorated digital mythology now surrounding the ability of SNSs to alter the mix and flow of ideas in media content, this study examines two core concepts that are crucial for the future of agenda-setting research: (1) the extent to which traditional media still lead (topically and over time) the public agenda as represented in SNSs, and (2) intermedia agenda setting within and across social media, particularly with attention to focusing events, sharing media, and creating original content.

On the first of these points, Sayre and his colleagues wrote that "...the rise of new media has the potential to result in a reverse flow of information. Particularly due to the speed with which many social media outlets such as YouTube and Twitter function, they may actually have the ability to influence the agenda of traditional news outlets" [21]. Though developing for decades, this potential is now further augmented by mobile devices that have the ability to not only immediately capture but also share breaking stories through social media $[32,33]$. Since most social media users in many countries can much more easily access and share information through social media outlets without much editorial or governmental oversight, when compared to traditional media, SNSs are now capable of not only breaking stories first but also building and setting traditional media agendas.

Yet when considering the different uses that are made of social networking sites, there is vastly less clarity on the extent to which certain social media lead (or follow) the agendas within other online social channels. While it is certainly not unusual for individuals to maintain active accounts on Twitter and Facebook, as well as to visit YouTube, Wikipedia, or any 
other online social media, the ways in which the residual content activity across different SNSs relates in terms of agendas has been understudied. Previous research typically focuses on only one social network site in relation to one traditional media outlet, and this practice has led to some conclusions where social media in general is treated as monolithic in its agenda-setting capacity [34]. Attention has also often only been given to one issue across agendas over time $[11,21]$ or a comparison of topics over both social and traditional media agendas $[28,29]$.

The study reported here has begun to close some of these gaps by first considering the topical agendas of two leading traditional media outlets (the New York Times and CNN) as well as the most common shared stories and trending topics on two popular SNSs (Facebook and Twitter). Time-series analyses have then been applied to the most prominent topics to track intermedia agenda setting across all outlets over time. This study is thus positioned to determine to what extent traditional media sets the agenda for social media, as well as the limits of reciprocity for the public's media agenda through SNSs to enter into the traditional media agenda. In addition, this study begins to compare intermedia agenda setting topically and across time within SNSs, and in so doing, adds a vital understanding of where traditional media, online uses, and content intersect around instances of focusing events, particularly elections.

This study thus examines four separate media agendas over a period of six weeks that strategically includes the 2010 US Midterm Election as an established focusing event. The influence of two distinct social media agendas and the underlying assumptions of media "produsage" [9] therein are thus examined against the flow of topics and investigation of subjects in traditional media. The following research questions were posed to examine reciprocity and the predictive capacity of SNSs in intermedia agenda setting.

\section{Research Questions}

RQ1: Which topics are made most salient in traditional media coverage, and which topics are made most salient on social networking sites?

RQ2a: Are there significant similarities between the topical agendas of traditional media channels and the agendas on social networking sites?

RQ2b: Are there significant similarities within the topical agendas on social networking sites?

RQ3a: On the most salient topics, do traditional media channels set the agendas for social networking sites?

RQ3b: On the most salient topics, do social networking sites set the agendas for traditional media channels?

RQ3c: On the most salient topics, does one social network site set the agenda for another social network site?

\section{Method}

The units of analysis in this study were headlines and trending items. The rationale for using these features to determine agendas is one that has been applied previously $[5,28]$. In addition, this analytical approach is also based on a rich background of work noting the critical importance of front matter, and headlines in particular, in attracting audience attention and facilitating shorthand interpretations of issues $[35,36]$. Though the distinction can be somewhat controversial in a converged media environment, for the purposes of this study, "traditional" media were represented by the New York Times and CNN. "Social" media, otherwise hypothesized as social networking sites, were represented here by two of the largest and most active, Facebook and Twitter.

The study thus began by collecting the top stories from the New York Times, CNN, Twitter, and Facebook for a period of six weeks in the fall of 2010 (11 October 2010 to 24 November 2010) to examine the research questions posed. The 2010 US Midterm Election of 2 November 2010 was specifically situated at the exact midpoint of this data collection period in order to observe coverage in various media around a known focusing event [37]. At randomly selected intervals (morning, afternoon, evening, early morning) each day during this timeframe, the online editions of the NYT [38] and CNN [39] were simultaneously captured, along with the most trended topics on Twitter and the most frequently shared stories on Facebook.

Trending topics from Twitter were gathered from http://whatthetrend.com [40] and filtered to only include the leading ten trending topic in the USA per day. Though unofficial, whatthetrend.com is self-defined as "the front page of the real-time web" and was particularly useful to categorize trends because for each trend, a brief audience-generated synopsis explains why it is trending. Most-shared stories on Facebook were pulled from http://itstrending.com [41] and limited to the eight most frequently shared headlines reported there [42]. In order to form more even comparisons with the news organizations NYT and CNN, items were filtered by the "News" categorization there. Though not officially endorsed by Facebook, itstrending.com operated by collecting all of the content shared via the Facebook Open Graph API and ranked each story, with complete headlines and original media outlet, solely by the number of times it was shared.

As with previous intermedia agenda-setting research [5], this study focused on what amounted to the "front pages" of the NYT and CNN online, which were subject to regular updating and had a clear hierarchy of most important items. These agendas were coded and then compared with more conceptual agendas of Twitter and Facebook that were determined by public activity in discussing or sharing certain topics within those spaces. Coding was completed by an individual trained in the categorical definitions with 
previous experience in content analysis. Preliminary coding was used to develop operational definitions for each category, minimize disagreements, and expand the codebook to include a "media" category. Following these negotiations, the coder independently made coding decisions for all of the headlines and trending topics in the sample.

The headlines and topics from each media outlet were categorized into one of 17 possible categories in an adapted codebook used previously in separate publications by Natarajan and Xiaoming [26] and Groshek [5]. The categories in these codebooks were: (1) Accidents/natural disasters, (2) Agriculture, (3) Business/economics, (4) Crime/criminal justice/law and order, (5) Ecology/environment, (6) Education, (7) Health care, (8) Military/national defense, (9) Politics, (10) Race/religion/culture, (11) Social problems/services, (12) Sports, (13) Technology, (14) War/terrorism, (15) Oddities, and (16) Undecided.

As already noted, this study added a (17) Media category. This decision was the result of preliminary coding that quickly revealed a regular amount of coverage about developments in media (i.e., Facebook, Twitter, YouTube, and other social media) or coverage of other topics in media, such as video games or forms of citizen journalism. Since this coverage did not fit neatly into existing categories-the "Technology" category, for example, was more about innovations such as new devices rather than media covering other media or media uses-the codebook was expanded on these grounds.

The reliability of the primary coder was determined by a second individual, otherwise uninvolved in the study, who coded a randomly selected $17.7 \%$ of the sample. Following some practice coding and a training session, intercoder reliability was 0.78 when calculated using Cohen's Kappa. Though coding was thus not free from error, and trending items on Twitter required additional training for both coders, this level of reliability with a measure that accounts for chance agreement was well above the minimum level of 0.70 indicated by Frey, Botan, and Kreps [43].

Once coded, the media agendas of these four outlets were determined by level of topic (category) salience and then rank-ordered, time-lagged, and compared to one another. Two key statistical techniques were applied to answer the research questions posed here. The first is a fairly common correlational measure of agreement among ordinal rankings, Spearman's rho, which is based on frequency of topical categories across media outlets. This ranking was derived simply by the number of times a headline topic was coded for each media outlet. For example, culture was the most common topic of headline coverage on CNN by raw frequency, with 103 instances $(22.9 \%)$ and for each media outlet, categories were ranked by frequency of appearance and then those rankings were compared across outlets for correlational association with Spearman's rho, which is a routine appropriate for such ordinal-level measures.
The second-Granger causality testing-is a timeseries analytical technique that has been growing in prominence in communication research, particularly with attention to agenda setting $[11,21,44,45]$. Though now discussed fairly regularly in literature, Granger causality calculates a significance test for measuring if the time lags of one variable (in this case, topic salience in a media outlet) relate to the distribution of another variable (here, topic salience in another media outlet) over time. Put somewhat briefly, Granger causality in intermedia agenda setting occurs when the distribution of topic salience in one media outlet explain a significant amount of variance of topic salience distribution in another media outlet, above the variance that can explained by endogenous topic salience time lags [46]. Compared to other time-ordered techniques, such as time-lagged correlations, and VAR or ARIMA modeling, Granger causality testing has been argued to be more accurate and provide clearer evidence of timeorder relationships $[11,47]$. In other words, this technique can statistically determine when topic salience in one media agenda precedes and predictively explains topic salience in another media agenda.

In order for Granger causality testing to be effective, however, several conditions must be considered. Data streams must have a minimum of 40 unique observations [48]; the six weeks of daily data coded for this study suitably has 45 instances. Variables must also achieve stationarity [49] to safeguard statistical validity; here, augmented Dickey-Fuller tests were conducted for each variable considered. All variables for each media outlet were uniformly transformed with a natural logarithm and all achieved stationarity. Finally, in Granger causality tests, an appropriate time lag must be selected. Though there is no conventional standard for determining time lags, most research relies on statistical criteria [50] derived for this purpose. This study utilized a minimum lag of one unit, which is required for Granger causality testing, and otherwise applied the most-identified suggested lag length across all lag criteria.

Of course, though Granger causality tests identify statistically significant time-ordered relationships between dyadic media agendas, they do not prove real-world causation $[51,21]$. Together with topical measures of media agendas, though, these analyses are very well suited to precisely examine reciprocity in intermedia agenda setting, specifically considering the predictive role of unique social networking sites.

\section{Findings}

1710 items were gathered over the six-week time frame identified here. Each headline and trending item was coded into only one of the categories from the codebook. For practical purposes, the lowest five categories accounted for no more than $1.6 \%$ of total units analyzed. These categories (Military, Social Problems, Education, Undecided, and Agriculture) were 
thus grouped together into a generic "Other" category that was not explicitly considered in terms of rankordering.

The first research question examined which topics are made most salient in traditional media coverage and which topics are made most salient on social networking sites. Table 1 summarizes the results observed here, where for CNN, NYT, and Facebook, the four most salient topics by category were: Culture, Politics, Crime, and Business. Though there was some variation across these media in terms of ranking the prominence of news items-bearing in mind that only the most shared news items on Facebook were tracked-a relatively high degree of similarity in topical agendas can be observed here between not only two traditional news agencies but also one social network site, Facebook.

Twitter, the other social network site considered in this study, demonstrated a much different agenda of most salient items. Specifically, the four most frequently identified categories on Twitter were (in order): Culture, Oddities, Sports, and Media. Thus, the topics made most salient varied across traditional to social media, at least with respect to the most talked-about trending topics on Twitter. Table 1 summarizes the rank-order of all topics by media outlet and the topical discrepancies there, most notably with regard to Twitter from all other outlets, can be readily observed.

Along these lines, RQ2a examined if there were significant similarities between the topical agendas of traditional media channels and the agendas on social networking sites. Here, it can be observed that the topical agendas of CNN and Facebook are highly correlated ( $p=0.85, p<0.01)$. When also measured with Spearman's rho, the New York Times' topical agenda is moderately correlated to that of the most frequently shared news stories on Facebook $(p=0.64$, $p<0.05$ ). Based on the findings shown in Table 1 , it is somewhat unsurprising to observe that the agenda of most popular trending topics on Twitter were not correlated with the agendas of either traditional media outlet. There was also no statistically significant relationship between the topical agendas of Facebook news shares and Twitter trending topics.

Thus, when considering RQ2a, there were differential relationships between traditional media and the two SNSs examined here. There was a fairly strong correlation between the topical agendas of both traditional media outlets and that of Facebook, but no relationship between any traditional media agenda modeled here and trending topics on Twitter. Likewise, analysis of RQ2b found no significant similarities within the topical agendas on the social networking sites Facebook and Twitter. In other words, the topical agenda of most trending items on Twitter were unrelated to any other media outlet over the time period analyzed here. Though not explicitly posed as a research question, it is worth reporting a moderately strong relationship $(p=$ $0.68, p<0.05$ ) between the topical agendas of the NYT and CNN. These results are summarized in Table 2.

Table 1. Issue salience by topical category in coverage on CNN, New York Times, Twitter and Facebook.

\begin{tabular}{lrrrrrrrr}
\hline Category & $\begin{array}{r}\text { CNN } \\
\text { Rank }\end{array}$ & $\begin{array}{c}\text { CNN } \\
\%\end{array}$ & $\begin{array}{c}\text { NYT } \\
\text { Rank }\end{array}$ & $\begin{array}{c}\text { NYT } \\
\%\end{array}$ & $\begin{array}{c}\text { Twitter } \\
\text { Rank }\end{array}$ & $\begin{array}{c}\text { Twitter } \\
\%\end{array}$ & $\begin{array}{c}\text { FB } \\
\text { Rank }\end{array}$ & $\begin{array}{c}\text { FB } \\
\%\end{array}$ \\
\hline Culture & 1 & 22.90 & 4 & 7.10 & 1 & 41.30 & 2 & 15.60 \\
Politics & 2 & 17.30 & 1 & 28.70 & 5 & 4.90 & 1 & 22.80 \\
Crime & 3 & 9.30 & 3 & 7.30 & 9 & 1.10 & 4 & 7.80 \\
Business & 4 & 7.10 & 2 & 20.20 & 8 & 1.60 & 3 & 9.70 \\
Accidents & 5 & 6.90 & 6 & 5.10 & 7 & 2.70 & 8 & 3.90 \\
Media & 6 & 5.80 & 9 & 3.10 & 4 & 5.30 & 5 & 7.20 \\
Health care & 7 & 5.60 & 8 & 4.00 & 11 & 0.70 & 6 & 6.70 \\
Oddities & 8 & 4.00 & 12 & 1.10 & 2 & 18.70 & 7 & 6.40 \\
Technology & 8 & 4.00 & 11 & 1.30 & 6 & 3.60 & 12 & 1.10 \\
Terrorism & 10 & 3.60 & 5 & 6.90 & 9 & 1.10 & 10 & 3.60 \\
Sports & 11 & 3.30 & 7 & 4.40 & 3 & 14.40 & 10 & 3.60 \\
Environment & 11 & 3.30 & 10 & 2.40 & 11 & 0.70 & 8 & 3.90 \\
Others & 13 & 6.90 & 13 & 8.20 & 13 & 4.00 & 13 & 7.80 \\
Total & & $100 \%$ & & $100 \%$ & & $100 \%$ & & $100 \%$ \\
\hline
\end{tabular}

Note: The four most frequent categories' rank order by media outlet appear in italics. 
Table 2. Bivariate Spearman's rho correlation matrix for issue salience by topical category in coverage on CNN, New York Times, Twitter and Facebook.

\begin{tabular}{llccc}
\hline & CNN & NYT & Twitter & Facebook \\
\hline CNN & -- & & & \\
NYT & $0.68^{*}$ & -- & & \\
Twitter & 0.24 & -0.07 & -- & \\
Facebook & $0.85^{* *}$ & $0.64 *$ & 0.18 & --
\end{tabular}

$* \mathrm{p}<0.05, * * \mathrm{p} \leq 0.01$

After considering the topical agendas of these traditional and social media outlets, this inquiry then proceeded to examine intermedia agenda setting of the most salient topics across different media outlets over time. To begin, RQ3a considered whether traditional media channels set the agendas for social networking sites on the most salient topics. The categories "Politics" and "Culture" were analyzed further as these were, on average, the two most prominent categories across all four media.

When looking at the distribution of political coverage over time, it can be observed that the frequency of political coverage on the New York Times Granger-caused political coverage $(X 2=4.35, p=0.02)$ on Twitter trending topics over time. In addition, the political coverage on CNN was shown to have Granger-caused (also with lags of two days) political coverage on Twitter, but only at $p<0.10(X 2=2.57, p=0.09)$. In terms of political coverage, there were no significant Granger-causal relationships between traditional media and Facebook over time. Though not explicitly entered as a research question, it is worth reporting that political coverage on the NYT Granger-caused $(X 2=3.65, p=0.036)$ the frequency of CNN's political coverage over time.

In examining coverage of culture, the frequency of such coverage on CNN was shown to have Grangercaused (with a lag of one day) cultural stories shared on Facebook ( $x 2=5.78, p<0.05)$. Likewise, the New York Times' cultural coverage frequency also Grangercaused this category of coverage $\left(X^{2}=3.55, \mathrm{p}=\right.$ 0.07 ) on Facebook but only at the $p<0.10$ threshold. There were no instances where the amount of cultural coverage in traditional media coverage was shown to have Granger-caused cultural trending items on Twitter, even though such coverage was the most regular type of coverage on Twitter by far.

Altogether, it can thus be observed that there are still fairly clear intermedia agenda-setting effects of traditional media on social networking sites, but that influence is not uniform across topics or the social media channels of Facebook and Twitter. Somewhat surprisingly, although uncorrelated topically across agendas, politics on Twitter was Granger-caused by both NYT and CNN political coverage as distributed over time. On the other hand, though Facebook was significantly correlated topically to traditional media agendas, its political coverage was not Granger- caused by either traditional network over time. However, cultural coverage on Facebook was actually Granger-caused by the frequency of such coverage in both the Times and on CNN.

As shown in Figures 1 and 2, there are clear differences in the distributions of political and cultural coverage over time and across media. Political coverage centers on a focusing event-the election of 2 November 2010-that appears to have shaped the frequency of coverage overall, but particularly on Twitter where almost no political coverage was observed before this date. Cultural coverage, on the other hand, followed a seemingly more regular cycle of coverage, such that the intermedia agenda-setting influence of traditional media could be observed only on Facebook.

The next research question (RQ3b) was posed to determine the extent to which social networking sites set the agendas for traditional media channels on the most salient topics. The two most dominant categories of coverage, politics and culture, were again modeled but in this case to measure potential reciprocity in intermedia agenda setting. Here, when looking at political coverage, Twitter (with a one-day lag) nearly Granger-caused politics coverage $(X 2=2.32, \mathrm{p}=0.11)$ on CNN, but only at a very generous level of statistical significance. There were no other predictive relationships regarding political coverage originating from SNSs to traditional media that approached statistical significance. Once more, though Facebook was more topically related to the agendas of both the New York Times and CNN, when analyzing the frequency of political stories shared on Facebook over time, there were no Grangercausal relationships that predicted political coverage in the NYT or on CNN.

When considering cultural coverage and the ability of social networking sites to set the agenda of traditional media over time, the frequency of cultural trending topics on Twitter did Granger-cause ( $x 2=6.11, \mathrm{p} \leq 0.05$ ) cultural coverage on CNN. No statistically significant relationships could be observed with the New York Times' coverage of cultural topics. The distribution of cultural news topics on Facebook was again unrelated, in the Granger-causal sense, to either traditional media outlet.

The intermedia agenda-setting effect of social networking sites during this period and across these media thus appears much more limited than that of traditional media [52], even as large numbers of users around the world create and share content through SNSs. In this study at least, there was only clear evidence that social media influenced the agenda of traditional media in the case of cultural trending topics on Twitter having Granger-caused cultural coverage on CNN. While Twitter also neared significance in Granger-causing political coverage on CNN, the distribution of cultural and political coverage in the New York Times was untouched by the amount of such coverage over time on the social networking sites studied here. The most frequently shared stories about these topics on Facebook had no predictive causal-type relationships whatsoever. 


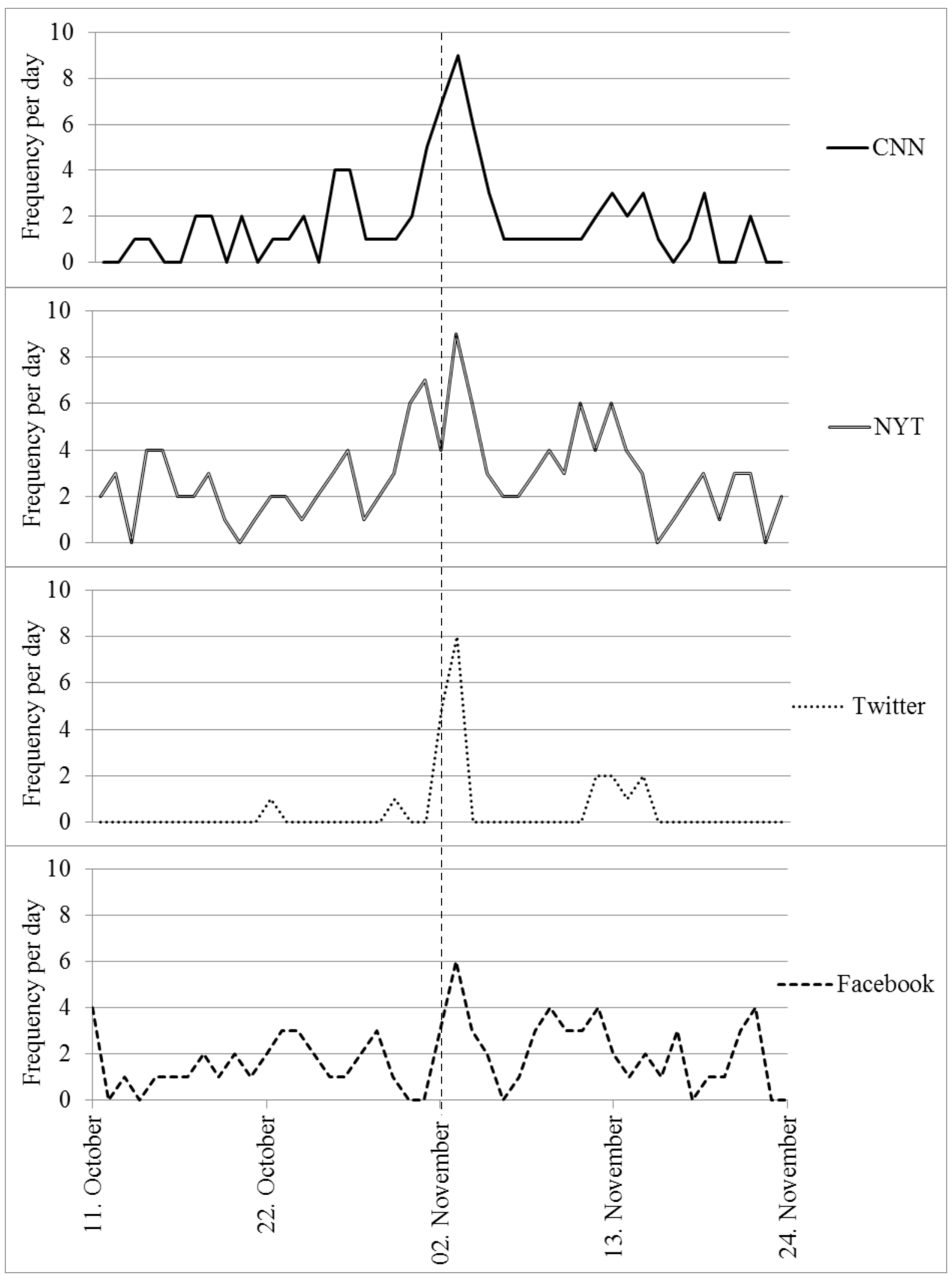

Figure 1. Political coverage over time across traditional media and social networking sites. Note: 2 November was the date of the 2010 US Midterm Elections. 


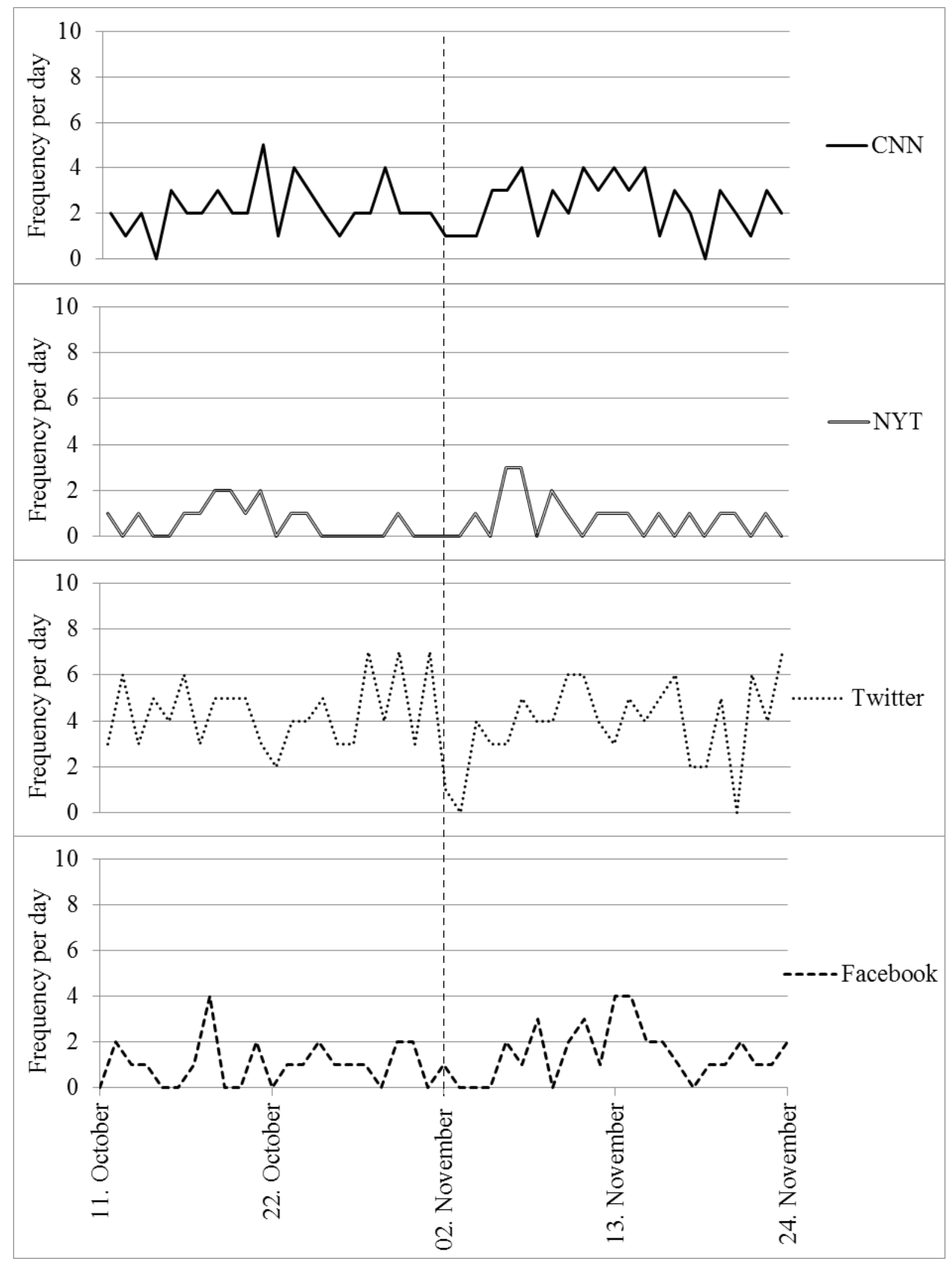

Figure 2. Cultural coverage over time across traditional media and social networking sites. Note: 2 November was the date of the 2010 US Midterm Elections. 
Further, when examining the last research question, there were no statistically significant Granger-causal relationships observed for political and cultural coverage within the social media analyzed in this study. RQ3c queried whether, on the most salient topics, one social networking site sets the agenda for another social networking site? Based on analyses performed here, the answer to that question is no-and there are by and large no topical or time-ordered relationships that can be observed within the agendas and the intermedia agenda-setting functions of social networks sites in this sample. The findings of all Granger causality testing are summarized in Table 3.

Table 3. Significant intermedia Granger-causal relationships in political and cultural coverage distributions across media.

\begin{tabular}{lcc}
\hline $\begin{array}{l}\text { Granger } \\
\text { Relationship }\end{array}$ & $\begin{array}{c}\text { Political } \\
\text { Coverage }\end{array}$ & $\begin{array}{c}\text { Cultural } \\
\text { Coverage }\end{array}$ \\
\hline CNN $\rightarrow$ Twitter & $2.57^{\#}$ & -- \\
CNN $\rightarrow$ Facebook & -- & $5.78^{*}$ \\
NYT $\rightarrow$ Twitter & $4.35^{*}$ & -- \\
NYT $\rightarrow$ Facebook & -- & $3.55^{\#}$ \\
CNN $\rightarrow$ NYT & -- & -- \\
NYT $\rightarrow$ CNN & $3.65^{*}$ & -- \\
Twitter $\rightarrow$ CNN & $2.32^{+}$ & $6.11^{*}$ \\
Twitter $\rightarrow$ NYT & -- & -- \\
Facebook $\rightarrow$ CNN & -- & -- \\
Facebook $\rightarrow$ NYT & -- & -- \\
Twitter $\rightarrow$ Facebook & -- & -- \\
Facebook $\rightarrow$ Twitter & -- & -- \\
\hline
\end{tabular}

$* p<0.05, \# p<0.10,+p<0.15$

\section{Conclusions}

During the timeframe and with the media analyzed here, it is evident that the public agenda-as manifest in trends and shares on social networking sites-has not yet come to drastically alter agendas of traditional media in a regularly predictive manner. While the distance from editors and journalists as gatekeepers of news and information flows to the public has clearly diminished with the popularization of social media $[53,54]$, it seems the potential for SNSs to directly shape media agendas does exist but only sporadically and on certain topics. Considering that different online media platforms, from blogs to various forms of social media-in this case Facebook and Twitter-allow for certain affordances and restrictions, it is reasonable to find that each platform demonstrated differential intermedia agenda setting potential as leveraged by the sociotechnical nature of its architecture.

The outward goals of this study were to more broadly examine intermedia agenda setting across topics and over time to examine if, and to what extent, different social networking sites could lead, rather than follow, the agendas of traditional media. To answer simply, yes, it is possible that a social networking site (Twitter) can predictively explain cultural coverage in a traditional media outlet (CNN), but apart from that finding, there is little concrete evidence of social media upending the shape and flow of news agendas. Indeed, as summarized in Table 3, there remains palpable evidence of traditional media setting the agenda in terms of both what was shared (Facebook) and what was created (Twitter) in social media spaces, though the level of measurement at the headline and categorical level does introduce limitations upon the analysis and its application to agenda setting at a finer gradation.

Still, while these findings are somewhat at odds with other research $[11,21,28,29]$, these other studies each often considered just one form of social media (commonly Twitter or YouTube) or focused on just one particular topic over time. The study reported here expands the scope of study and makes a useful contribution by identifying both topics that generate the most attention and become most salient in social networking sites, as well as making comparisons of intermedia agenda setting between multiple traditional media outlets and within social media. Results observed here indicate that not all social media are created equal with regard to both agendas and influence - but also that events, particularly the ones that can be predicted, like elections, are especially pertinent in shaping social media agendas.

In particular, findings from this study suggest that topically, Facebook is relatively strongly related to both CNN and the NYT in terms of topic salience. Comparatively, trending topics on Twitter are not significantly related to the topical agendas of either traditional media or another social media outlet. Yet, when set in a predictive capacity on specific topics, trending topics on Twitter can actually precede and help explain traditional media (CNN) coverage of culture-and nearly show the same relationship (where $p=0.11$ ) with political coverage (again on CNN). The distribution of most frequently shared political and cultural stories on Facebook, however, did not show any predictive Granger-causal relationships over time, despite being far more closely related to the topical agendas of both traditional media outlets examined here.

Thus, while there is a tendency in both popular and academic literature toward grouping social networking sites as somewhat similar entities in terms of their transformative effect $[55,56]$, it is worth noting the activities and uses that take shape in these spaces can be much different [22]. These unique uses can, of course, lead to unanticipated outcomes $[13,14,51]$, particularly with regard to agenda setting. While there is good reason to note the growing import and influence of user-generated culture across media industries, caution should also be exercised to delimit forms of social media uses, often structured by the social networking sites themselves to make better sense in tracking the influence each does or can have in larger online or offline settings [57].

On the other hand, traditional media more consist- 
ently set the agendas in time-ordered Granger-causal capacity for both Twitter and Facebook. Somewhat interestingly, these relationships were also bound to certain social networking sites by topics. Political trending topics were Granger-caused by traditional media on Twitter and shared cultural stories were Granger-caused by traditional media on Facebook. Parsing out exactly which casual mechanism explains these findings of why one and not the other is difficult and not readily apparent by looking at content alone. Other scholars, such as Huberman, Romero, and Wu [58] have identified proximity and anonymity as important features that discriminate uses of online media, and Kwak, Lee, Park and Moon [29] found that less reciprocity between users functionally situates Twitter as closer to mass media in that relatively few senders produce most of the news for an audience of followers.

Altogether, while the precise rationale for why politics on Twitter and culture on Facebook were Grangercaused by traditional media is not readily apparent, these results nonetheless clearly suggest that intermedia agenda setting needs to be considered in more circumspect terms-directionally, topically, and with respect to precisely identified media outlets. As the results of this study further indicate, it is certainly important to consider social media in intermedia agenda-setting processes because certain social networking sites have the potential to shape elite agendas, and in this study cultural coverage on Twitter was shown to Granger-cause cultural coverage on CNN. We generally characterize this outcome as agenda trending and it suggests that social media trends can, indeed, set traditional media agendas. Yet while the findings noted here suggest that agenda trending is taking place, it seems only at cetain moments where the impact of social media can key in to focus on an event [59].

While it is speculative as to why cultural coverage on CNN was predicted by cultural coverage Twitter and cultural coverage on the NY Times was not, one potential explanation may be the regular integration of Twitter in particular on normal CNN broadcast coverage, which is a feature certainly less shared by the NYT. Notably, the content patterns observed in the agendas of social media were shown here to have been informed by those very same elite agendas, or likewise to extend the agendas of traditional media further.

In this study, the 2010 US Midterm Elections was situated within data collection as an expected, fixed focusing event. Under this circumstance, traditional media was shown to have primarily led social media coverage of politics, with the exception of Twitter trends showing a very limited $(p<0.15)$ Grangercausal relationship to political coverage on CNN. On balance, the importance of focusing events-whether previously identifiable or emergent, as in the case of cultural coverage on Twitter-seems to be a key feature of intermedia agenda setting that has been somewhat overlooked in previous literature $[60,61]$. As shown here, patterns of agenda reinforcement with relatively limited reciprocity and innovation can still be observed in the contemporary user-producer media environment.

While some scholars [10] have suggested the end of agenda setting may be in sight, this study offers some empirical evidence of the adaptability and amplification of traditional media agendas through the public in social media trends. In addition, findings presented here signal the reciprocal capacity of social media in intermedia agenda setting, specifically in instances where topical similarities are limited. These findings also identify a pressing need for further specificity in not only clarifying intermedia agenda-setting flows through certain social media, but also additional attention to focusing events and their topics that may well facilitate more evenly reciprocal agenda-setting processes, such as those described by Meraz [11]. Although the results reported here do not precisely align with previous work on blogs, they do not necessarily conflict, and rather work to extend and add nuance to the conceptualization of SNSs as the next iteration of online media that may well contribute to a shaping of traditional media agendas as the field of gatekeepers continues to widen by producers and deepen by influence.

Practically speaking, the differential levels of agenda-setting influence observed here suggest Twitter is more likely to follow, rather than lead, political agendas formed by traditional media and cultural coverage on Facebook is more clearly set by agendas on traditional media. Conversely, cultural coverage on Twitter was the one category of coverage where a social media channel set the agenda for a traditional one, in this case CNN. On a theoretical level, these findings open up a space where agendas must be considered not only topically-where there may be little apparent relationship-but also temporally, where within-topic flows may suggest greater agenda-setting reciprocity over time and across channels. Considered jointly, the outcomes of this study consequently identify that the nature, structure, uses, and content of Facebook and Twitter are unique but still compatible and possibly complementary in the public arena, particularly as each is differently shaped and potentially shaping traditional media agendas. 


\section{References}

1. McCombs M, Shaw D. The Agenda-setting Function of the Mass Media. Public Opinion Quarterly. 1972; 36(2):176-185.

2. Weaver DH. Audience Need for Orientation and Media Effects. Communication Research. 1980;7(3): 361-373.

3. Ghanem S. Filling in the tapestry: The second level of agenda setting. In: McCombs MD, Shaw DL, Weaver $\mathrm{DH}$, editors. Communication and Democracy: Exploring the Intellectual Frontiers in Agenda-Setting Theory. Mahwah, NJ, USA: LEA; 1997. pp. 3-14.

4. McCombs M. Setting the agenda: The mass media and public opinion. Malden, MA, USA: Blackwell Publishing; 2004.

5. Groshek J. Homogenous Agendas, Disparate Frames: CNN and CNN International Coverage Online. Journal of Broadcasting and Electronic Media. 2008; 52(1):52-68.

6. Song Y. Internet news media and issue development: A case study on the roles of independent online news services as agenda-builders for anti-US protests in South Korea. New Media Society. 2007;9(1): 71-92.

7. Roberts M, Wanta W, Dzwo T. Agenda setting and issue salience online. Communication Research. 2002;29(4):452-465.

8. Papacharissi Z. The virtual sphere. New Media \& Society. 2002;4(1):9-27.

9. Bruns A. Blogs, Wikipedia, Second Life, and beyond: From production to produsage. New York, NY, USA: Peter Lang; 2009.

10. Johnson TJ. The Abacus, 8-Track Tapes...and Agenda Setting? Available from: http://mediaconvergen ce.org/blog/?p=1054 (accessed on 10 December 2010)

11. Meraz S. Using Time Series Analysis to Measure Intermedia Agenda-Setting Influence in Traditional Media and Political Blog Networks. Journalism \& Mass Communication Quarterly. 2011;88(1):176-194.

12. Entman R. Cascading activation: Contesting the White House's frame after $9 / 11$. Political Communication. 2003;20(4):415-432.

13. Baum MA, Groeling T. Online media and the polarization of American political discourse. Political Communication. 2008;25(4):345-365.

14. Boczkowski PJ. de Santos M. When more media equals less news: Patterns of content homogenization in Argentina's leading print and online newspapers. Political Communication. 2007;24(2):167-180.

15. Reese SD. Theorizing a globalized journalism. In: Loeffelholz M, Weaver $\mathrm{DH}$, editors. Global journalism research: Theories, methods, findings, future. London, UK: Blackwell; 2008. pp. 240-252.

16. Gans H. Democracy and the news. New York, NY, USA: Oxford University Press; 2003.

17. boyd dm, Ellison NB. Social network sites: Definition, history, and scholarship. Journal of Computer-Mediated Communication. 2007;13(1), article 11.
18. Bennett $\mathrm{WL}$, Iyengar $\mathrm{S}$. A new era of minimal effects? The changing foundations of political communication. Journal of Communication. 2008;58(4):707731.

19. Kaufhold K, Valenzuela S, Gil de Zúñiga H. Effects of citizen and professional journalism on political knowledge and participation. Journalism \& Mass Communication Quarterly. 2010;87(3/4):515-529.

20. Maier S. All the News Fit to Post? Comparing News Content on the Web to Newspapers, Television, and Radio. Journalism and Mass Communication Quarterly. 2010;87(3/4):548-562.

21. Sayre B, Bode L, Shah D, Wilcox D, Shah C. Agenda Setting in a Digital Age: Tracking Attention to California Proposition 8 in Social Media, Online News, and Conventional News. Policy \& Internet. 2010;2(2): 7-32.

22. Papacharissi Z. A private sphere: Democracy in a digital age. Cambridge, UK: Polity Press; 2010.

23. Ragas MW, Kiousis S. Intermedia Agenda-Setting and Political Activism: MoveOn.org and the 2008 Presidential Election. Mass Communication and Society. 2010;13(5):560-583.

24. Pfetsch B, Adam S. Media Agenda Building in Online and Offline Media-Comparing Issues and Countries. Proceedings of the 6th ECPR General Conference, Reykjavik, Iceland, 25-27 August 2011.

25. Kaufmann C. Threat inflation and the failure of the marketplace of ideas: The selling of the Iraq war. International Security. 2004;29(1):5-48.

26. Natarajan K, Xiaoming H. An Asian Voice? A Comparative Study of Channel News Asia and CNN. Journal of Communication. 2003;53(2):300-314.

27. Plasser F. From hard to soft news standards? How political journalists in different media systems evaluate the shifting quality of news. The Harvard International Journal of Press/Politics. 2005;10(2):47-68.

28. Zhao WX, Jiang J, Weng J, He J, Lim E, Yan H, Li X. Comparing Twitter and Traditional Media using Topic Models. Proceedings of the 33rd European Conference on Advances in Information Retrieval, Dublin, Ireland, 18-21 April 2011.

29. Kwak H, Lee C, Park H, Moon S. What Is Twitter, a Social Network or News Media? Proceedings of the 19th International Conference on World Wide Web, Raleigh, NC, USA, 26-30 April 2010.

30. Birkland TA. Focusing Events, Mobilization, and Agenda Setting. Journal of Public Policy. 1998;18(1): 53-74.

31. Bennett LW, Livingston S. Gatekeepeing, Indexing, and Live-Event News: Is Technology Altering the Construction of News? Political Communication. 2003; 20(4):363-380.

32. Hermida A. From TV to Twitter: How Ambient News Became Ambient Journalism. M/C Journal. 2010; 13(2).

33. Williams A, Wardle C, Wahl Jorgenson K. "Have they got news for us?" Audience revolution or business as usual? Journalism Practice. 2011;5(1):85-99. 
34. Swigger N. The Online Citizen: Is Social Media Changing Citizens' Beliefs about Democratic Values? Political Behavior. 2013;35(3):589-603.

35. Yang J. Framing the NATO air strikes on Kosovo across countries: Comparison of Chinese and US newspaper coverage. International Communication Gazette. 2003;65(3):231-249.

36. Pan Z, Kosicki GM. Framing analysis: An approach to news discourse. Political Communication. 1993;10(1):55-75.

37. Walker LD, Waterman RW. Elections as Focusing Events: Explaining Attitudes toward the Police and the Government in Comparative Perspective. Law \& Society Review. 2008;42(2):337-366.

38. New York Times. Available from: http://www.ny times.com (accessed on 11 October 2010 until 24 November 2010).

39. Cable News Network. Available from: http://us. cnn.com (accessed on 11 October 2010 until 24 November 2010).

40. What the Trend. Available from: http://whatthe trend.com (accessed on 11 October 2010 until 24 November 2010).

41. Its Trending. Available from: http://itstrending.com (accessed on 11 October 2010 until 24 November 2010).

42. Sometime after data collection, itstrending.com has gone offline, at least temporarily as of writing. This situation does not diminish the effectiveness of itstrending as an unbiased aggregator of Facebook shares.

43. Frey L, Botan C, Kreps G. Investigating communication: An introduction to research methods. 2nd ed. Needham Heights, MA, USA: Allyn \& Bacon; 2000.

44. Groshek J. Media, instability, and democracy: Examining the Granger-causal relationships of 122 Countries from 1946 to 2003. Journal of Communication. 2011;61(6):1161-1182.

45. Tan Y, Weaver DH. Agenda-Setting Effects among the Media, the Public, and Congress, 19462004. Journalism \& Mass Communication Quarterly. 2007;84(4):729-744.

46. Enders W. Applied economics time-series. Hoboken, NJ, USA: John Wiley \& Sons; 2004.

47. Stock JH, Watson MW. Introduction to econometrics. Boston, MA, USA: Addison; 2003.

48. Poole MS, McPhee RD, Canary, DJ. Hypothesis testing and modeling perspectives on inquiry. In: Knapp $\mathrm{ML}$, Day JA, editors. Handbook of interpersonal communication. Thousand Oaks, CA, USA: Sage; 2002. pp. 23-72.

49. Stationarity is defined as a quality of a process in which the statistical parameters (such as mean and standard deviation) of the process do not change with time.
50. These criteria can be derived using the VARSOC command in STATA and include final prediction error (FPE), Akaike's information criterion (AIC), Hannan and Quinn information criterion (HQIC), and Schwarz's Bayesian information criterion (SBIC).

51. Atukeren E. Christmas cards, Easter bunnies, and Granger-causality. Quality \& Quantity. 2008;42(6): 835-844.

52. In a series of sub-analyses, at $p<0.05$, election-specific coverage on Twitter was shown to predict election coverage on NYT ( $X 2=3.45, p \leq 0.05)$, but all other results, including the reciprocal NYT Grangercausing election coverage on Twitter $(X 2=5.90, p \leq$ 0.05 ) were from traditional to social media, where CNN Granger-caused election coverage on Twitter ( $\mathrm{x} 2$ $=5.63, p \leq 0.05)$ and CNN also Granger-caused election coverage on Facebook $(x 2=6.27, \mathrm{p} \leq .05)$. This finding reiterates the need for further modeling of agenda setting at both topical and temporal levels, with attention to examining (rather than extrapolating) a variety of social media outlets.

53. Groshek J, Dimitrova D. A Cross Section of Political Involvement, Partisanship and Online Media in Middle America during the 2008 Presidential Campaign. Atlantic Journal of Communication. 2013;21(2):108-124.

54. Gurevitch M, Coleman S, Blumler JG. Political Communication-Old and New Media Relationships. The ANNALS of the American Academy of Political and Social Science. 2009;625(1):164-181.

55. Boulianne S. Does Internet Use Affect Engagement? A Meta-Analysis of Research. Political Communication. 2009;26(2):193-211.

56. Zhang W, Johnson TJ, Seltzer T, Bichard SL. The revolution will be networked: The influence of social networking sites on political attitudes and behavior. Social Science Computer Review. 2010;28(1):75-92.

57. Deuze M. Media Life. Cambridge, UK: Polity Press; 2012.

58. Huberman BA, Romero DM, Fang W. Social networks that matter: Twitter under the microscope. First Monday. 2009;14(1).

59. Asur S, Huberman BA. Predicting the Future with Social Media. HP Labs Working Paper. Available from: ar xiv.org/pdf/1003.5699 (accessed on 10 December 2011).

60 . Cobb R, Elder C. The politics of agenda-building: An alternative perspective for modern democratic theory. Journal of Politics. 1971;33(4):892-915.

61. Walgrave S, Van Aelst P. The Contingency of the Mass Media's Political Agenda Setting Power: Toward a Preliminary Theory. Journal of Communication. 2006;56(1):88-109. 